\title{
A Case of Encephalopathy in an Immunocompetent Adult with Persistent Parvovirus B19 Viremia
}

\author{
Ariel Antezana ${ }^{1, *}$, Ilya Kister ${ }^{2}$ and Joseph Herbert ${ }^{2}$ \\ ${ }^{I}$ Neuromedical Clinic of Central Louisiana, Alexandria, LA, USA \\ ${ }^{I}$ NYU School of Medicine, New York, NY, USA
}

\begin{abstract}
Parvovirus B19 (PVB19) is a ubiquitous airborne virus; it is well known to cause erythema infectiosum (fifth disease) in children. Neurologic complications after PVB19 exposure are thought to be transitory and infrequent in the adult population. Herein, we report an interesting case of a young woman who developed cognitive deficits, white matter changes and persisting viremia for over a year after exposure to PVB19.
\end{abstract}

Keywords: Cognitive deficits, encephalopathy, parvovirus B19, white matter changes,

\section{CLINICAL PRESENTATION}

A previously healthy 33 year-old woman developed cognitive deficits, dizziness, arthralgias and myalgias a few days after her daughter was diagnosed with Fifth disease (parvovirus B19, PVB19). She had no fever, headache and menginsums. The patient was referred for neurologic consultation after her cognitive symptoms persisted and she was fired from office job. Neurologic exam was unremarkable, but neuropsychological testing confirmed a variety of cognitive deficits, predominantly in areas of speed of processing, attention, working and verbal memory. Timeline of disease course, treatments, testing is shown in Fig. (1).

MRI of the brain demonstrated small juxtacortical, subcortical and pontine non-enhancing hyperintensities on T2/FLAIR, which increased in number of a follow up study (Fig. 2). Initial cerebrospinal fluid (CSF) studies were remarkable for elevated leukocyte count of 12 cell $/ \mathrm{ml}^{\wedge} 3$, with lymphocyte predominance, but no oligoclonal bands (OCBs). Repeat CSF studies showed normalization of cell count, 2 OCBs and borderline IgG index of 0.67 (normal 0.66). A six-week course of Ceftriaxone was given by infectious disease specialist for suspected Lyme's disease, followed by a course of intravenous Methylprednisolone for presumptive neuro-inflammatory process. Neither intervention yielded an observable benefit.

In view of parvovirus exposure, serum antibodies against PVB19 were tested and found to be elevated: $\mathrm{IgG}$ of 4.6 (normal 0-0.8) and IgM of 1.0 (normal 0-0.8). PVB19 Polymerase Chain Reaction (PCR) in serum demonstrated 9800 copies $/ \mathrm{ml}$ nine months after onset, which decreased to 2000 copies $/ \mathrm{ml}$ at one-year follow up. Diagnosis of parvovirus-induced encephalopathy [2] was suggested and a

*Address correspondence to this author at the Neuromedical Clinic of Central Louisiana, Alexandria, LA, USA;

E-mail: antezana.neuromed@gmail.com course of Intravenous Immunoglobulin (IVIG) $0.4 \mathrm{~g} / \mathrm{Kg}$ for 5 days was administered and monthly thereafter for 6 months. The treatment resulted in subjective improvement in malaise and arthralgias, but not cognition. PVB19 viremia resolved at 2-year follow-up, but she continued to report cognitive, vestibular and sensory symptoms.

\section{DISCUSSION}

PVB19 is a ubiquitous airborne virus that can infect people of all ages, but mostly children. PVB19 infection is typically asymptomatic, but can cause erythema infectiosum ('slapped cheek disease'), arthropathy, and, much less commonly, transient aplastic crisis, persistent anemia and hydrops fetalis. Severity of the disease depends, in part, on the immunologic status of the host with grave cases of aplastic crisis, pure red cell anemia and death in immunodeficient patients lacking neutralizing antibodies [1]. Neurological complications after PVB19 infection have been described; a recent systematic review of neurological manifestations of PVB19 reports encephalitis, encephalopathy and meningoencephalitis represent $63.3 \%$ of all PVB19 CNS related cases; several of them $68 \%$ were in immunocompetent patients [2,3]. Permanent neurologic damage with cognitive deficits is also described and presumed to be immune-mediated [4]. We present a case of a young immunocompetent adult with encephalopathy and persistent PVB19 viremia for at least 12 months following parvoviral infection. Differential diagnosis of encephalopathy and white matter lesions on MRI includes mainly infectious causes such as Lyme's disease, viral (Cytomegalovirus, Varicella, Herpes, West Nile virus) [5-7], immune-mediated diseases (multiple sclerosis, sarcoidosis, vasculitis), toxic-metabolic (CO exposure, B12 deficiency), vascular etiologies. Our patient underwent extensive work up to rule out these etiologies. Neurodegenerative disorders were considered unlikely given acute onset of symptoms, patient's age and lack of progression. The diagnosis of 


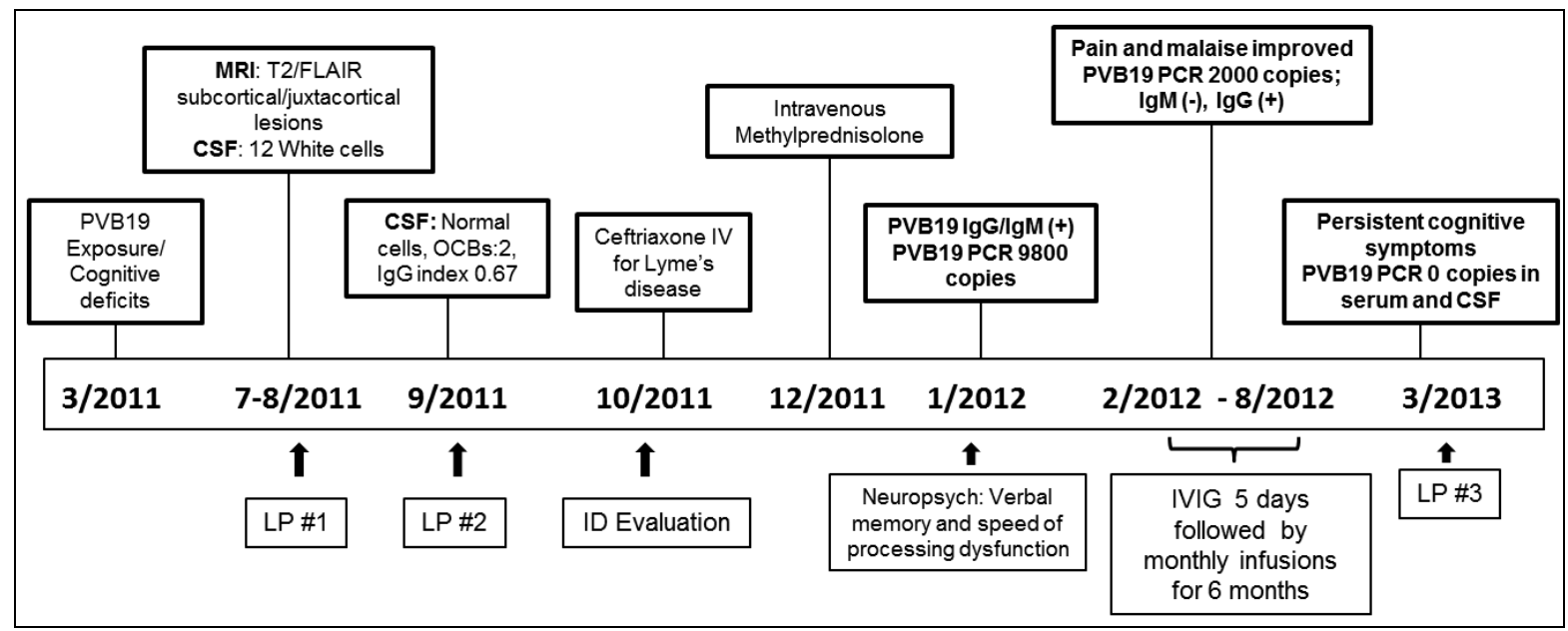

PVB19: Parvovirus B19; MRI: Magnetic Resonance Imaging; FLAIR: Fluid Attenuated Inversion Recovered; LP: Lumbar Puncture, CSF: Cerebro-spinal fluid, OCB: Oligoclonal Bands, ID: Infectious disease; PCR: Polymerase Chain Reaction; IVIG: Intravenous Immunoglobulin.

Fig. (1). Timeline of disease course and treatments.
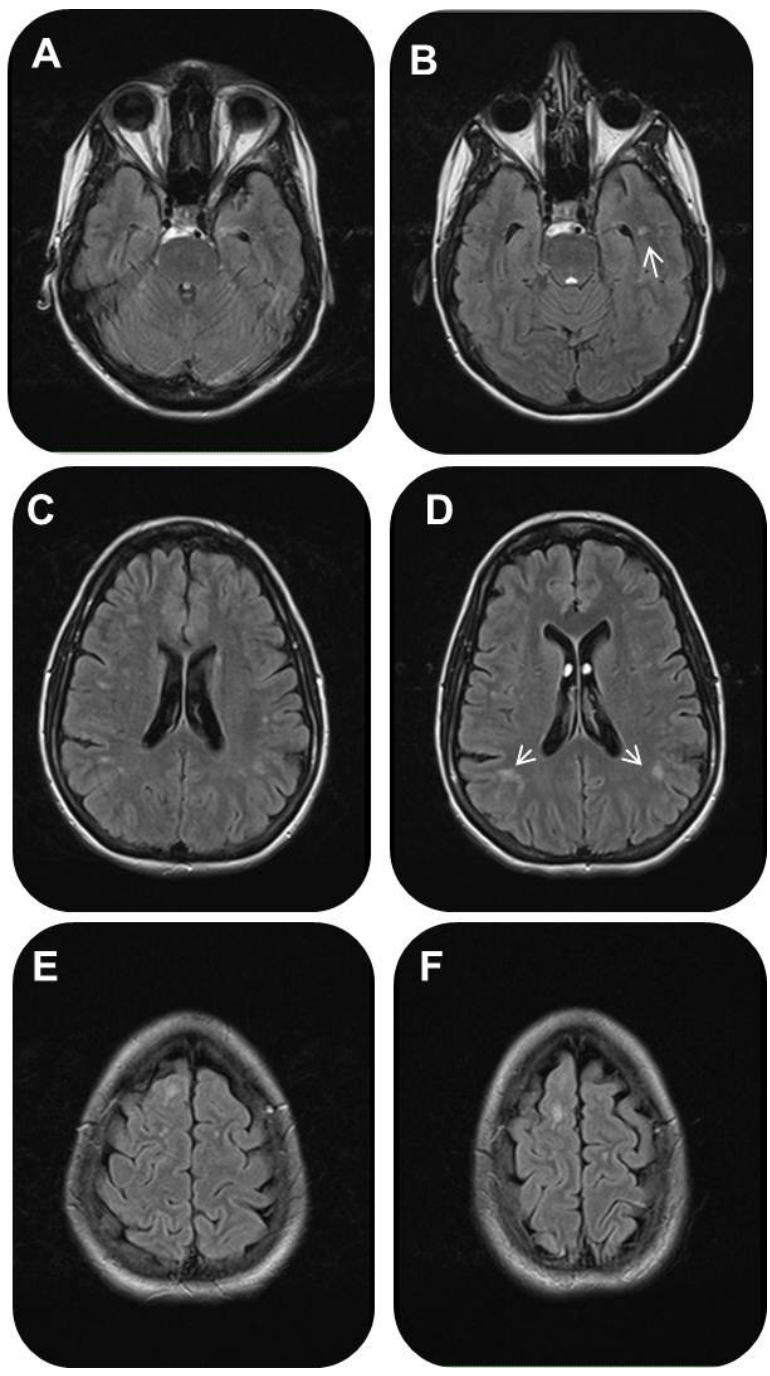

Magnetic Resonance Images: A,C and E initial MRI which showed Subcortical and juxtacortical white matter lesions on FLAIR images. B,D and $\mathrm{F}$ two months follow up MRI showing few more white matter lesions (arrows).

Fig. (2). Brain MRI. 
PVB19- associated encephalopathy is supported by clinical history, elevation of $\operatorname{IgM}, \operatorname{IgG}$ antibodies [3] in the acute phase and remarkable persistence of PVB19 in serum for a year after exposure.

Systemic parvoviral infection can last from 3-198 days $[1,3]$ with mean duration of 55 days. There is no specific treatment for PVB19 and the majority of cases resolve spontaneously; severe cases of aplastic crisis or pure red cell anemia can respond to IVIG $[1,2,4]$. However, as our case demonstrates cognitive deficits, which may be related to increasing burden of subcortical hyperintensities on brain MRI, can persistent for years after exposure despite treatment with IVIG. We recommend that patients with neurologic symptoms following PVB19 infection should have serologic and spinal fluid examinations for PVB19 IgG, IgM and PCR as well as MRI of the brain. IVIG could be considered for neurogically-impaired patients with persistent PVB19 viremia. A systematic study of neurologic sequella of PV19 infection and their treatment is warranted.

\section{CONFLICT OF INTEREST}

The authors report no conflict of interest related to this work.

\section{ACKNOWLEDGEMENTS}

No funding was received for the publication of this work.

\section{REFERENCES}

[1] Neal S, Young MD, Kevin E, Brown MD. Mechanism of disease parvovirus B19. N Engl J Med 2004; 350: 586-97.

[2] Bara F, Whiteside S, Batista S, Morris J. Neurological aspects of human parvovirus B19 infection: a systemic review. Rev Med Virol 2014; 24(3): 154-68.

[3] Miltiadis D, Nathan L, Goldman DL, Neurologic manifestations associated with parvovirus B19 infection. Clin Infect Dis 2009; 48: 1713-23.

[4] Kerr JR, Barah F, Chiswick ML, et al. Evidence for the role of demyelination, HLA-DR alleles, and cytokines in the pathogenesis of parvovirus B19 meningoencephalitis and its sequelae. J Neurol Neurosurg Psychiatry 2002; 73: 739-46.

[5] Arciniegas D, Anderson A. Viral encephalitis: neuropsychiatric and neurobehavioral aspects. Curr Psychiatry Rep 2006; 6(5): 40-6.

[6] Heegaard ED, Brown KE. Human parvovirus B19. Clin Microbiol Rev 2002; 15(3): 485-505.

[7] Fujimoto H, Asaoka K, Imaizumi T, Ayabe M, Shoji H, Kaji M Epstein-barr virus infections of the central nervous system. Intern Med 2003; 42: 3340. 